\title{
Statistical Analysis of Measurements in Exact and Inexact Sciences: An Open Problem
}

\author{
Lia Queiroz do Amaral \\ amaral@if.usp.br \\ Instituto de Física, Universidade de São Paulo, Brazil
}

\begin{abstract}
Differences between statistical analysis of measurements in exact and inexact sciences are the focus of this work. The early and independent beginning of Probability and Statistics had a theoretical synthesis, with an initial development based in Physics and Astronomy. This lead to Error Theory, used in Statistics of Measurements in Exact sciences, with defined criteria of validity. This direction of Mathematical Physics resulted in the progresses and achievements in Classical Physics, and also on established ways of treating measurements of physical properties. It is discussed that Exact Sciences treat only Inanimate Matter, and things that can be defined and measured, in terms of only seven fundamental physical quantities, with the definition of the International System of Units (SI). On the other hand a direction of Mathematical Statistics emerged later on, based on "Sampling", to study properties of a population, with criteria of significance, within validity intervals, which depend on the size and characteristics of the studied sample, and on the inferences to be made in the research. These are two very different approaches, but both use probability density functions related to hypothesis about data. The modern inferential sampling statistics can be applied to all practical problems, in particular in Biology and Humanities, where there are "models", but not Theories as in Physics. The word "theory" is many times used in a mistaken way. Life and Human Sciences use this modern type of Statistics. This paper discusses a particular case, in which the same ensemble of experimental results in samples of biological origin (hairs from hominoids) can be analyzed with the two different statistical approaches, in a proposal for Human Evolution, and the conditions for inference of accurate conclusions are discussed. A philosophical discussion between subjective and objective criteria of the researcher is made, and also of the concept of knowledge.
\end{abstract}

Keywords: probability, statistics, measurements, error theory, sampling, human evolution.

\section{Introduction}

Probability and Statistics had different origins in Ancient Times. Statistics deals with data treatment and inferences, from information on demography and economics of a Political State. Probability deals with likelihood, plausible opinion on processes governed by chance (gambling and dice games, chance and uncertainty, random processes). These two different directions started to be unified conceptually and theoretically much later on, with an interesting history [1].

The developments in the $19^{\text {th }}$ century deal with Randomness and Uncertainties, in several different directions. The integration of statistics with Physics led to basic analytical tools for mathematical physics and also to experimental procedures to measure physical properties. Theory of errors evolved with the introduction of the method of least squares, published by the French mathematician Adrien-Marie Legendre in 1805. The French mathematical physicist and astronomer Pierre-Simon Laplace publishes his "Analytic Theory of Probability" (1812) and works on his five volumes on Celestial Mechanics, defining the basis of classical mechanics. The focus is on probability density functions giving hypothesis on data, able to represent frequency curves of experimental physical measurements. The other giant of the period is the German mathematical physicist and astronomer Carl Friedrich Gauss, whom besides the normal Gaussian distribution function turned also to problems in Magnetism. The direction of Mathematical Physics results in the progresses and achievements in Classical Physics, and also on established ways of treating measurements of physical properties. Probability found also a major new application in physical science, the theory of gases, which developed into statistical mechanics, with reflexes in Chemistry, defined as a Science with the establishment of the periodic table of elements, universally recognized by 1890. The interaction between Physics and Statistics was really defined in function of use of Statistical Physics in Thermodynamics, to deal with the problem of molecules, as discussed by James Clerk Maxwell in 1873 [2]. 
In another direction statistics develops as an independent branch of Mathematics, with broad applied interests, and the English statistical school is defined, with the Statistical Society of London founded in 1834, and the ISI- International Statistical Institute established in 1885.

The beginning of the 20th century saw the integration of Physics with Chemistry, forming the exact sciences, with a complex history [3].

This paper focus on a pragmatic open problem: Error Theory in Exact Sciences Versus Sampling Statistics in Life and Human Sciences. The Scientific Method in Exact Sciences evolves with theories on the subject of study and on forms of measuring properties of the subject of study. The error theory as learned in Graduate courses in Physics is based on the idea of existence of a true value, and how measurements can approach it. Errors may be systematic, or random, due to the apparatus used for measurements and the uncertainties inherent to the measure procedure. Errors propagate through equations used to compare experimental results with theories, and there are defined criteria for the interval of validity. The Error Theory is usually presented to graduate students in the form of Laboratory manuals, and an old book in English has a suitable presentation [4].

On the other hand, Sampling Statistics used in Biology and Human Sciences correspond to Studies of characteristics of a population, through analysis of a "sample" of the population. There are Statistical criteria of significance and sample definition, depending on sample size and characteristics, and on the questions to be made in the research. The sampling statistics was defined in the beginning of the 20th century, in connection with the English school of statistics.

These two approaches are indeed very different, presenting philosophical and pragmatic problems, as further discussed in the next items of this paper. After discussion of the two existing approaches of Statistics, and their problems, a new model for Human Evolution [5] is discussed, in terms of of the two statistical approaches.

\section{Measurements in Exact Sciences}

Every process of measurement depends on instruments, and there are limits of precision. The quantitative result of a measurement is represented by "significant algharisms", together with the accepted precision. Due to the inherent imprecision of measurements, they must be repeated, following statistical criteria, in order to obtain average values (as estimates of the "true value") and other parameters. An ensemble of independent measurements is then analyzed according to the well known statistical criteria (histograms and graphics with the frequency distribution). This is the basic logics, valid for a single researcher, or by a large number of measurements performed by several independent researchers. Such large ensemble of results leads to the concept of "BIG DATA", used in artificial intelligence (AI, a subject outside the scope of this article). It should be considered that the logics of statistical treatment depend on WHAT is being measured, and on the conclusions and inferences to be extracted from the measurements. It is at this point that there is a fundamental difference between physical sciences (called "exact") and the treatments used in biology and human sciences. Such differences seem to not have been fully recognized, neither by scientists nor by lay people, and this is the focus of the present paper.

To measure is, by definition, to compare with a standard. The definition of standards started with the French Revolution (decimal system), and is made by the International Bureau of Weights and Measures (BIPM), in Paris, created on 20 May 1875, following the signing of the Treaty of the Metre. It started with basic standards used in Mechanics (space, time and mass), and along history it came to the now well defined seven fundamental physical quantities, with the definition of the International System of Units (SI), established in 1960. Physicists and chemists reached then a historical agreement, with the use of the isotope Carbon 12 for unity of mass. Metrology is defined by the BIPM as the science of measurement, embracing both experimental and theoretical determinations at any level of uncertainty in any field of science and technology. The main facts related to the SI system can be found in [6].

There are only seven fundamental physical quantities that are measured in physical fundamental units: time (second); length (metre), mass (kilogram), electric current (ampere), thermodynamic temperature (kelvin), amount of substance (mole), luminous intensity (candela). All the other unities are derived from these 7 basic units, by defined equations, forming a coherent system, which serves also Engineering and commerce.

Along history, the standards artifacts have changed from physical objects (the metre, the kilogram) and time fraction of the year (sec) to more abstract and exact standards. The metre has been the first to be redefined, in terms of wavelength of the spectral line of krypton 86, a universal natural phenomenon, in the definition of the SI. In 1967 the sec is redefined in terms of the frequency transition between two levels of the atom of Cesium 133. In 1983 the metre is redefined in terms 
of the sec, using the value of the velocity of light. Since 1999 studies started in order to redefine the kilogram. Today the 7 initial physical quantities have been replaced by 7 "natural constants", or numbers.

Such evolution meant the passage from physical concepts, related to physical experiments and measurements, to the corresponding theories, were the universal constants could be defined. So, physical sciences started with concepts, possible to be defined and measured, and arrive to numbers, through the exact quantification of the measurements.

Mathematical statistics uses the concept of "variance" while in physics it is more used "standard deviation" (square root of the variance). It should be stressed that there exists always an evaluation of the errors, made by the scientist, both in data acquisition and in its interpretation. Many times the precision is given with only one figure. It is the criterion of the scientist (or of the group of scientists) that validates both the data and its interpretation. There is not automatic objectivity in Natural Sciences.

A fundamental point to emphasize is that the exact sciences (physics and chemistry) deal only with physical quantities (and properties) possible to define and measure. This means that exact sciences deal only with inanimate matter. Error Theory and Statistics of Measurements are used in the study of known objects, meaning Samples with known atoms. Atoms of a given type are considered identical. Properties of an ensemble of atoms depend on controlled parameters in the measurement:

- temperature, pressure, applied fields (electric, magnetic), chemical composition of the sample

These systems follow statistics of measurements and error theory. There is a problem on studies of "natural materials", since traditional Journals of Chemistry require very well defined samples. Studies with "natural materials" are therefore very difficult to publish, since there are many uncontrolled variables.

These criteria are not followed in the more pragmatic Sampling Statistics which became "the" Statistics of all disciplines in the 20th century.

\section{Inferential Sampling Statistics}

The Inferential Sampling Statistics used in several "inexact" sciences, correspond to the study of characteristics (not necessarily well defined "physical quantities") from a population, through the analysis of a "sample" of the population. This direction was defined in the 20th century, especially by the English school of Statistics. A key person in this direction was Karl Pearson, statistician and applied mathematician who introduced Biometry and made his career at University College London, with the method of moments and the chi square test. After him came Sir Ronald Aylmer Fisher, British statistician, who developed the analysis of variance (ANOVA), and also geneticist with theories of sexual selection, following Darwin's '. The basic of this direction is in his book of 1925 [7].

It is important to focus on "Student", the pen name of William Sealy Gosset, a brewer of Guinness in Dublin and the pioneer in the analysis of small samples, who was a student of Pearson and had much interaction with Fisher, after publication of his paper defining the $t$ distribution in 1908 [8]

It should be stressed that Engineering is not an "exact science", it uses scientific knowledge for human interests, and is subjected to rules and laws of industry and commerce. The importance of Student's distribution in all branches of applied science can be seen in [9].

The basic difference is that Error Theory, used in exact measurements, is valid only for the 7 fundamental physical quantities, serving to test Theories in Physics and Astronomy, related to inanimate matter. The modern inferential sampling statistics can be applied to all practical problems, in particular in Biology and Humanities, where there are "models", but not Theories as in Physics. It is necessary to emphasize that the word "theory" is many times used in a mistaken way. The lay people in general do not know the differences between hypothesis, model, Theory and Law of Nature, words with very different meaning in Exact Sciences and in Humanities, and even in Life Sciences. It requires a discussion in Philosophy, since facts, hypotheses, laws, and theories are separate parts of the scientific method.

There are theories from first principles (ab initio) satisfying criteria of mathematical logics, and phenomenological theories, focused in explaining experimental results. There is also a difference between the study of closed systems, with control of entrance and exit, possible of theoretical modeling, and open systems, where classical thermodynamics is no longer valid. This means that not all physical theories are able to explain a complex reality, with too many variables in action. Helmer and Rescher [10] discussed the epistemology of the inexact sciences and questioned the mythology of exactness, emphasizing that in several areas of applied natural science decisions, predictions, and explanations are made only after exact procedures are mixed with unformalized expertise. 
A recent article testing models as hypotheses in the inexact sciences [11] focus epistemic uncertainties (resulting from lack of knowledge). The proposed approach allows taking these epistemic uncertainties into account in a pragmatic way before any model runs are made.

The proposal for human evolution in this paper follows such 'pragmatic realist' philosophy. The question: Is there an exact science for Life? has no easy answer. In fact, Life exists, independently of a Theory that could explain it. It is well established that physical laws apply to living organisms, but such laws are unable to predict the emergence of Life. Molecular Biology developed enormously since the discovery of DNA structure, when Rosalind Franklin and Maurice Wilkins, using X ray crystallography, revealed in 1952 the helical structure of the DNA molecule, and James Watson and Francis Crick realized that DNA was made of two chains of nucleotide pairs, that encoded the genetic information for all living things. Watson, Crick, and Wilkins received the Nobel Prize in 1962, and Rosalind (already dead) was not even mentioned. The history of the union of molecular biology with genetics and biochemistry can be followed in [12].

It must be stressed here that, when samples have known chemical composition, their measurements in Biochemistry and Biophysics academic studies may follow the statistics of Errors used in exact sciences. However, experiments made with "biological material" must follow instead Inferential Sampling Statistics, inclusive with use of "control samples" for comparison and analyzes of specific effects. In particular Medicine was empirical from its very beginning, and cannot be classified as an Exact Science. The frontiers of Medicine have strong connections with biomedical industry, and it is possible to say that academic knowledge in Molecular Biology will eventually come together with medical research in the future, but that has not been achieved yet.

In Humanities there exists not even a scientific basis of consensus. The only alternative at this moment is to focus on the biological evolution leading to humans. This is the direction of the next item of this paper.

\section{A new proposal for Human Evolution}

Darwin's theory of evolution is now considered the only basis for Biology, but it is not in fact a Theory in the sense of Physical Theories. And the neo-Darwinism has cut down the central proposals of Darwin, who proposed originally the existence of two processes for biological evolution, a "natural selection for adaptation to the ambient" and a "sexual selection" defining the forms of reproduction, giving descendents [13].

Human Evolution is only treated by Darwin in terms of sexual selection. He gives great importance to the nudity of human skin, different from the other primates, and also to human vocalizations, sexually differentiated, inclusive because vocalizations among apes occur in the context of sexual signaling and disputes. Darwin's original proposal did not receive acceptance in his time, particularly regarding the role of female sexuality and "female choice". The modern synthesis, in the beginning of the 20th century, unifying genetics with natural selection, kept only Darwin' s proposal of "competition among males", but just ignored Darwin' s proposal regarding "female choice", common among animals. Sexual selection has been treated by Ronald Fisher in the context of "runaway process" [14].

Sexual selection is nowadays studied in the evolution of several biological species, but not with the same focus as given by Darwin in human evolution. It is time for a revision, to reach some understanding about Human Evolution.

This work proposes analysis of an ensemble of results relevant to the biological evolution from the ape ancestral to the human lineage, using criteria from Physics and Biology. The basic idea is very simple, can be said in few words:

- From the biological differences with the other apes, the human species can be classified as "biped without body fur", and these characteristics emerged millions of years before the increase of the brain. All Apes from our lineage carry their infants clinging to the body fur of their mothers, an essential function for infant survival. The mechanical analysis of infant carrying and of the physical properties of Ape hairs [5] shows that fur reduction brings necessarily the biped locomotion, as a direct consequence of the selective pressure of mothers carrying their infant in their arms.

There is a decreasing scale law among primates, relating the $\log$ of the relative density of hairs (hairs/cm 2 divided by the total surface area) with the log of total surface area $(\mathrm{cm} 2)$. The small gibbon has ten times more hair density than the large gorilla, and about 30 times less weight than the Gorilla. Gibbon infants are safely carried, clinging to the mother's hairs, while the Gorilla newborn requires that the mother hold it with one hand, walking tripedally for some months, until the infant is able to adopt the mounting position. The properties of hominoid hairs were studied in detail in [5], and are here discussed. Three pieces of skin with hairs, each with about $200 \mathrm{~cm} 2$ area, came from 3 individuals, from 3 different species of Apes: 1 gibbon, 1 orangutan and 1 gorilla (chimpanzee was not available). The 3 samples are very different, easily recognized by their appearance and texture, typical of each species. Gibbon hairs are black, thin and smooth silky, 
those from orangutan are reddish, thicker and rough, while those of gorilla are black with intermediate thickness and texture. Even if the samples come from a single individual of each species, the hairs can be classified as "known sample", from all that has been studied by forensic analysis of hairs.

The mechanical properties of natural fibers are defined by the central cortex, made of keratin, and depend on the area of the transversal section of the fiber, so that the research can be made with relatively few hairs. The elasticity and the resistance capacity of the hairs is measured in stress/strain curves, one for each hair. Curves give the deformation (given as a fraction of the original elongation) due to the applied force (given in Newtons), until the breakage of the individual hair. Ten hairs of each sample of the 3 individuals have been measured, and the results are displayed in figure 1, together with the result for human head hair, for comparison. In order to publish the paper all statistical principles used in statistics of small samples, that is Student's method and ANOVA comparisons, were performed. With such statistical analysis the paper was published, and figure 1 shows the stress strain curves, discussed after it.

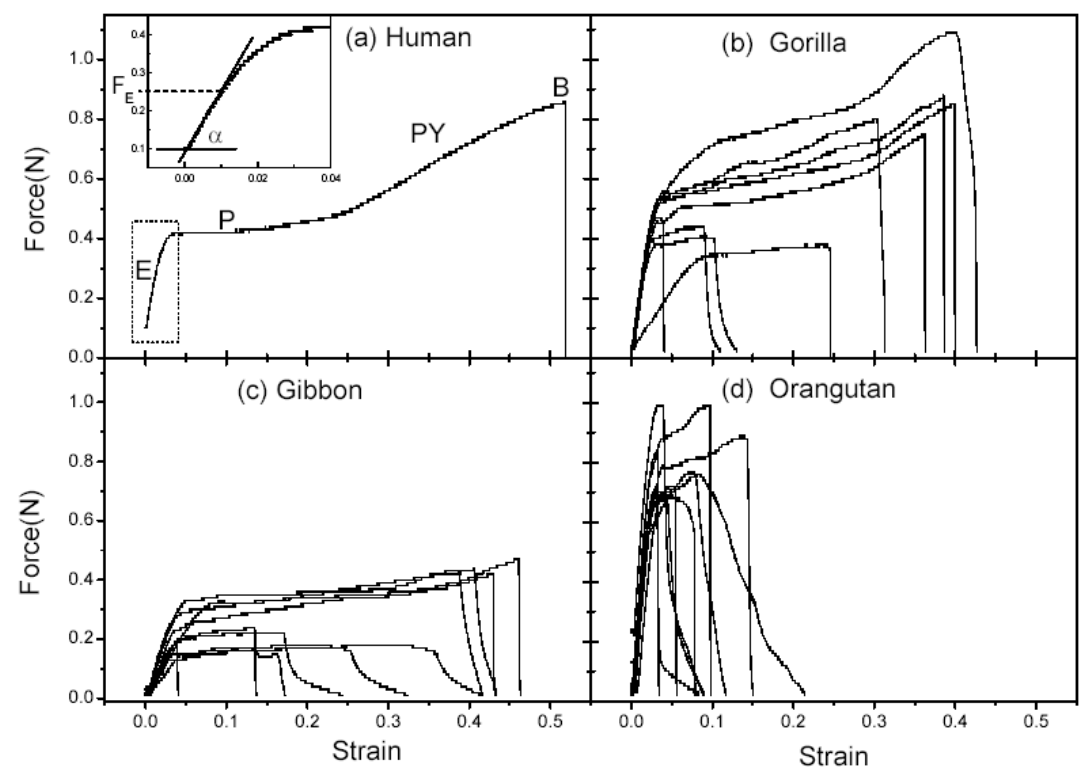

Figure 1 - reproduction of figure 2 from [5]

Stress-strain curves for Ape hairs: (a) human, (b) gorilla, (c) gibbon and (d) orangutan. In (a) E corresponds to the linear region, P to the plastic plateau, PY to the "pos-yield" region, and B to the break of the hair. The insertion in (a) is an amplification of the linear region, permitting to obtain the elastic force $F_{E}$ and the linear coefficient $\alpha$.

There is an initial elastic region, following a linear Hooke law, until about $1 \%$ deformation (elastic limit $\mathrm{F}_{\mathrm{E}}$ ), followed by a plastic region, due to changes in the conformation of the keratin molecule, which is influenced by the amount of water, and a final break of the hair. Curves are typical of all natural fibres, and in the human hair reveal the treatment given to it. The regression coefficient of the initial linear region $(\alpha)$ gives the Young modulus of elasticity $Y$, in units of pressure $\left(\mathrm{GPa}=109 \mathrm{~N} / \mathrm{m}^{2}\right)$, through the relation $\mathrm{Y}=\alpha / \mathrm{A}$, where A is the area of the cross section of the hair, $\varnothing$ its diameter, so that $\mathrm{A}=\pi(\varnothing / 2)^{2}$. The elastic limit $\mathrm{F}_{\mathrm{E}}$ is the limit that the hair can stand, in order to be able to support the pressure given by infant clinging to the hair. From each curve it is possible to measure the values of $\alpha$ and $F_{E}$ (as shown in the insert in the figure), and also the values at the rupture point ( $\mathrm{Fr}$ e $\mathrm{Sr}$ ). The force to rip out the hair from the skin has also been measured (20 hairs in each sample) and it is a little larger than the elastic limit.

Figure 1 shows the evident differences between the samples from the different species. The conventional statistical analysis, used in biology, has been made and is given in the paper [5], since hairs are a "natural biological sample". Differences between species are statistically significant, in the comparison of each pair of species, and also in the Anova test for the 3 ape species. The paper [5] has an extensive discussion on the implications of the results regarding safety of infant carrying along our evolutionary line. Also tests showed that a handful with 100 hairs is necessary to support infants 
with few kilograms, an estimate consistent with the quantity of hairs that the infant hand can grasp while clinging to the mother's hairs.

What is done here now are some extra considerations, with a more simplified analysis of the results, displayed in Table 1, constructed from data presented in [5]. A comparison with analysis of the same data using simple Error Theory used in statistics of measurements in exact sciences is attempted, with the focus on extracting ideas that can give new hints on the process of Biological Evolution towards humans, along phylogenetic evolution of the Hominoid branch where humans are inserted.

\section{Table 1 - constructed from data of [5]}

Thickness of the skin and length of hair (estimated) average values of the measured values of the hair diameter $\varnothing$, of the linear regression coefficient $\alpha$, of the elastic limit $F_{E}$, and from Young modulus Y. Averages show the standard deviation of the mean, in parentheses the coefficient $\mathrm{CV}=100 \mathrm{x}$ standard deviation / average value. Final lines show the weight of the adult animals and the density of hairs of each species.

\begin{tabular}{|c|c|c|c|c|}
\hline & GORILLA & GIBBON & ORANGUTAN & HUMAN \\
\hline $\begin{array}{l}\text { Skin }(\mathrm{mm}) \\
\text { thickness }\end{array}$ & $\sim 1.5$ & $\sim 0.6$ & $\sim 2.3$ & \\
\hline $\begin{array}{c}\text { Hair } \\
\text { Length }(\mathrm{cm})\end{array}$ & $\sim 6$ & $\sim 4$ & $\sim 10$ & \\
\hline$\varnothing(\mu \mathrm{m})$ & $66 \pm 2(21 \%)$ & $52 \pm 2(19 \%)$ & $120 \pm 4(24 \%)$ & $\begin{array}{c}60-80 \\
\text { ethnic determined }\end{array}$ \\
\hline$\alpha(\mathrm{N})$ & $17 \pm 2(25 \%)$ & $6.0 \pm 0.6(33 \%)$ & $32 \pm 1(13 \%)$ & \\
\hline$F_{E}(N)$ & $0.38 \pm 0.03(30 \%)$ & $0.18 \pm 0.02(36 \%)$ & $0.49 \pm 0.02(12 \%)$ & $0.28 \pm 0.02(29 \%)$ \\
\hline $\mathrm{Y}(\mathrm{GPa})$ & $5.0 \pm 0.6(42 \%)$ & $2.8 \pm 0.3(43 \%)$ & $2.8 \pm 0.2(36 \%)$ & $2.9 \pm 0.2(33 \%)$ \\
\hline Weight Adult (Kg) & $150-250$ & 7 & $50-90$ & \\
\hline $\begin{array}{l}\text { Hair Density } \\
\text { hairs } / \mathrm{cm}^{2}\end{array}$ & 100 & 1000 & $\sim 200$ & \\
\hline
\end{tabular}

From the obtained results, Hominoid phylogenetic evolution can be rationalized in terms of reproduction / survival of the infants, as sketched in Figure 2.

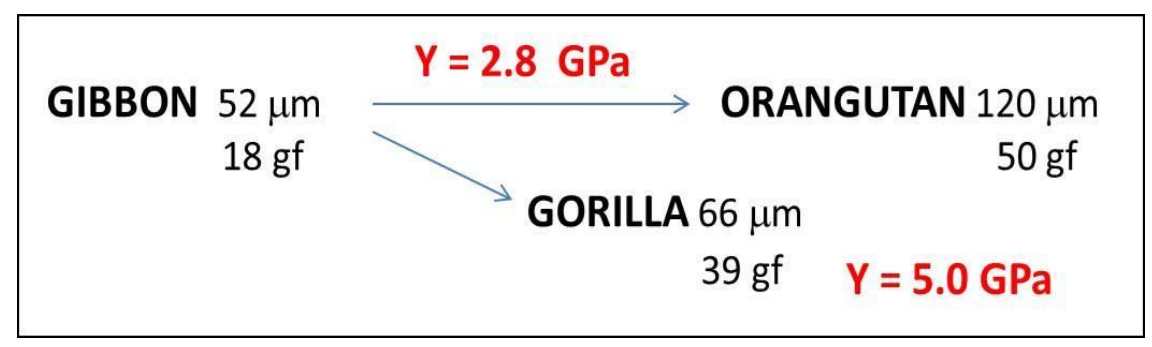

Figure 2 - Sketch showing average values of the hair diamete $\varnothing(\mu \mathrm{m})$, the elastic limit $\mathrm{F}_{\mathrm{E}}(\mathrm{gf})$, and the Young modulus $\mathrm{Y}(\mathrm{GPa})$ along Asian evolution (gibbon to orangutan) and African line to Gorilla.

Biology usually requires a "sample of the population", and results obtained from only 3 individuals, one of each species, is considered "unusual", and also that only 10 hairs have been measured for each individual. But in physical sciences the research is usually made in one sample of a known material, with repetition of measurements of a physical property. So that 10 independent measurements of one individual may represent a sufficient ensemble, since it is known for 
sure that the individual is from a specific ape species. Such reasoning may be compared with identification of individuals by DNA analysis. Extra information enters the statistical ensemble of data, since it is known and accepted that the 3 individuals represent each one a separate species.

In physics it is not usual the use of statistical analysis in terms of the parameter "t of student" as function of the degree of freedom and confidence level. Usually analysis uses simply Gaussian function, when applicable, since the probability of the distance to the average value depends on the standard deviation $\sigma$, in a known way:

- $68.2 \%$; $95.4 \%$ and $99.7 \%$ probability for the measure to be within respectively $1 \sigma, 2 \sigma$ and $3 \sigma$ from the average value. The same inference can be made here using simpler data treatment usual in Physics, since there is enough separation between the values of all measured parameters for the different species.

In order to place these results on hominoid hairs in the context of human evolution, some further information [5] must be given:

-The hominoid line evolved along the last ca. 20 million years ago, and has very few living species: first in Asia the small gibbon, followed by the bigger Orangutan, and later in Africa the very big Gorilla, followed by the chimpanzee and humans together.

-Humans cannot be placed in the primate scale law regarding fur, the denudation process is unexplained, but chimpanzee already deviates, having the same density of hairs as gorillas, but being much smaller, with weight about $1 / 3$ of the gorilla.

-There is a pattern in the evolution of hominoid socio-sexual structures, of not easy explanation: Gibbons are small and monogamous, Orangutans are solitary and Gorillas have harém, but none of them accept coexistence with other males. Growth in size, together with large sexual dimorphism, indicates sexual selection among Orangutan and Gorilla. Chimpanzees are promiscuous, males are genetically related in their groups, while Humans are flexible. The whole process of initial separation of humans from the ape lineage is uknown.

Taken into account all the available information, it is possible to infer a pattern about evolution, from the obtained results of hairs of the three species. The increase in size and weight in the Asian line occurs with the same young modulus $\mathrm{Y}$, but with increase in hair diameter $\emptyset(\mu \mathrm{m})$ and elastic limit Fe (gf) from Gibbon to Orangutan. The African direction, with the much heavier Gorilla, required a larger value of the Young modulus Y, allowing for not so large increase in hair diameter $\emptyset(\mu \mathrm{m})$ and elastic limit Fe (gf). Hair physical properties might have evolved correlated to the pressure of safe infant carrying. On the other hand, the increase in size, together with the large sexual dimorphism correlates with sexual selection. Further inferences on human evolution are outside the scope of this work, focused on the statistics used in the analysis of properties of hominoid hairs.

This work brings some basic philosophical questions regarding scientific knowledge, and the role of the scientist in the subjective search for "meaning". The dichotomy male/female implies very complexes mating systems, with logical inconsistency between sexes, of very difficult solution. Mathematical and statistical methods are important for advance of knowledge, but the inner consistency of assumptions and integration with information outside data are essential. The logic of this work joins justified qualitative arguments, together with solid quantitative results.

\section{Conclusion}

It must be stressed that this work did not "prove" the view that infant carrying is a most important variable in Human Evolution. But it has the merit to show new directions of research, from analysis of defined mechanical problems, not properly taken into account previously. Physical ideas and concepts, formulated according to intuitive logics, may be very helpful in the analysis of complex problems, such as Human Evolution.

Physics advanced through experiences intended to answer a theoretical question, based on assumptions of the theory. Thus exact science get away from complexities of nature, studying only controlled situations, simulating the accepted assumptions. Physics is therefore dependent on advances in mathematics which open new directions for exact knowledge. But it must be remembered that mathematical logics are based on axioms, self-evident, taken for granted as true. It seems clear that knowledge on subjects still outside exact sciences (life and humans) can advance only through openings due to new intuitions. 


\section{References}

[1] F. N. (Florence Nightingale) David, Games, gods and gambling : the origins and history of probability and statistical ideas from the earliest times to the Newtonian era. New York, Hafner Publishing Company, 1962

[2] O. Rey. "How statistics entered physics?". 7th International Conference of the European Society for the History of Science, Czech Society for the History of Sciences and Technology; Charles University, Prague, Czech Republic. Sep 2016, HAL-01662284.

[3] K. Gavroglu and A. Simões, Neither Physics nor Chemistry - A History of Quantum Chemistry. Cambridge, MA, MIT Press, 2012. Reviewed by Alán Aspuru-Guzik, Physics Today vol. 65, no. 8, 52-54, 2012.

[4] Y. Beers. Introduction to the Theory of Error. London, Addison-Wesley, second edition, 1957.

[5] L. Q. Amaral, "Mechanical analysis of infant carrying in hominoids", Naturwissenschaften vol. 95, no. 4, pp. 281292, 2008.

[6] J de Boer, "On the History of Quantity Calculus and the International System", Metrologia vol. 31, pp. 405-429, 1995.

[7] R. A. Fisher, Statistical Methods for Research Workers. Edinburgh, Oliver \& Boyd, 1925.

[8] Student, "The probable error of a mean", Biometrika, vol. 6, no. 1, pp. 1-25, 1908.

[9] S. T. Ziliak, "Retrospectives Guinnessometrics: The Economic Foundation of "Student's" t", Journal of Economic Perspectives, Vol. 22, No. 4, pp. 199-216, 2008.

[10] O. Helmer, N. Rescher, "On the epistemology of the inexact sciences", Rand Corporation, Report No. P-1513, Santa Monica, CA, 1958

[11] K. Beven, "Towards a methodology for testing models as hypotheses in the inexact sciences", Proc. R. Soc. A 475: 20180862, 2019.

[12] M. Morange, A History of Molecular Biology, translated by Mattew Cobb, Cambridge, Harvard University Press, 1998.

[13] C. Darwin. The descent of Man and Selection in relation to sex. London, John Murray 1st ed. (2 volumes), 1871.

[14] R.A. Fisher, "The evolution of sexual preference", Eugenics Review vol. 7, no. 3, pp. 184-192, 1915 